\title{
PCI Planning Strategies for Long Term Evolution Networks
}

\author{
Hakan Kavlak $^{1}$ and Hakki Ilk ${ }^{2}$ \\ ${ }^{1}$ RAN Network Consulting, \\ Ericsson Japan K.K, Tokyo, Japan \\ hakan.kavlak@ericsson.com \\ ${ }^{2}$ Ankara University, Faculty of Engineering, Electronics Engineering Department, \\ Tandogan, Ankara, Turkey \\ ilk@ankara.edu.tr
}

\begin{abstract}
In Long Term Evolution (LTE) networks, physical cell identity allocation (PCI) is crucial for quality of service and somewhat similar to scrambling code allocation in WCDMA. PCI, or Layer 1 identity, is an essential configuration parameter of a radio cell. It identifies the cell in mobility functions such as cell reselection and handover. In this paper simulation results, in order to optimize PCI planning in LTE networks is developed and several recommendations for PCI planning strategies are presented.
\end{abstract}

Keywords: Network planning, Optimization, LTE, PCI Planning.

\section{Introduction}

As smartphone penetration increase all over the world, mobile users consume more data and wish for faster networks. From speech only GSM to LTE, this demand has to be satisfied. Today, an ordinary LTE network can reach up to $100 \mathrm{Mbit} / \mathrm{s}$. In order to reach this kind of speed, LTE networks should be well optimized. As an initial step; optimization and design part of LTE defines the name of the cells so that users can camp on the best server cell and handover the other cells while the user is moving [3]. One of the basic functions in any network is the cell search. During this procedure, time and frequency synchronization are established between the user equipment (UE) and the network. To identify the cells, the Physical layer Cell Identity (PCI) is acquired. This is achieved by the cell search procedure [1], [8]. The Physical Cell Identity, or Layer 1 identity, is an essential configuration parameter of a radio cell. It identifies the cell in mobility functions such as cell reselection and handover. The PCI is also used to determine the location of the resource elements containing the Physical Control Format Indicator Channel (PCFICH) and Physical HARQ (Hybrid automatic repeat request) Indicator Channel (PHICH) [8]. If cells` PCI cannot be assigned well, mobile users cannot read the actual signals, cannot camp on the LTE networks or data throughput is degraded even worse, it is dropped. That means high cost network becomes useless [13]. PCI consists of two signals; Primary Synchronization Signal 
(PSS) and the Secondary Synchronization Signal (SSS). The detection of these two signals not only enables time and frequency synchronization, but also provides the UE with the physical layer identity of the cell and the cyclic prefix (CP) length, and informs to the UE [2]. For Cell Search procedure UE first detect PSS and then SSS as shown in Fig 1.

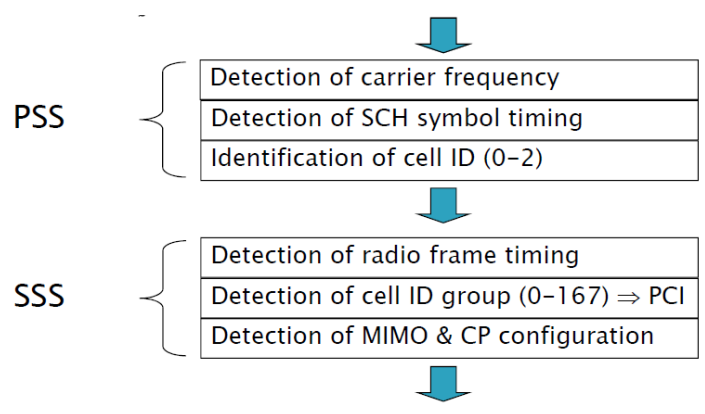

Fig. 1. Cell search precedure

The primary sequence is referred to as the Primary Synchronization Signal. Three PSS sequences are used in LTE, corresponding to the three physical layer identities within each group of cells to estimate $5 \mathrm{msec}$ timing and physical-layer identity. It is used to determine S-SCH (synchronization channel) symbol timing. [11] 168 pseudo random sequences represent the Secondary Synchronization Signals. They determine frame timing and the cell identity group. Each SSS sequence is constructed by interleaving, in the frequency-domain, two length-31 BPSK-modulated secondary synchronization codes, denoted here $\mathrm{m}_{0}$ and $\mathrm{m}_{1}$. Frame structure of PSS and SSS can be shown in Fig 2.

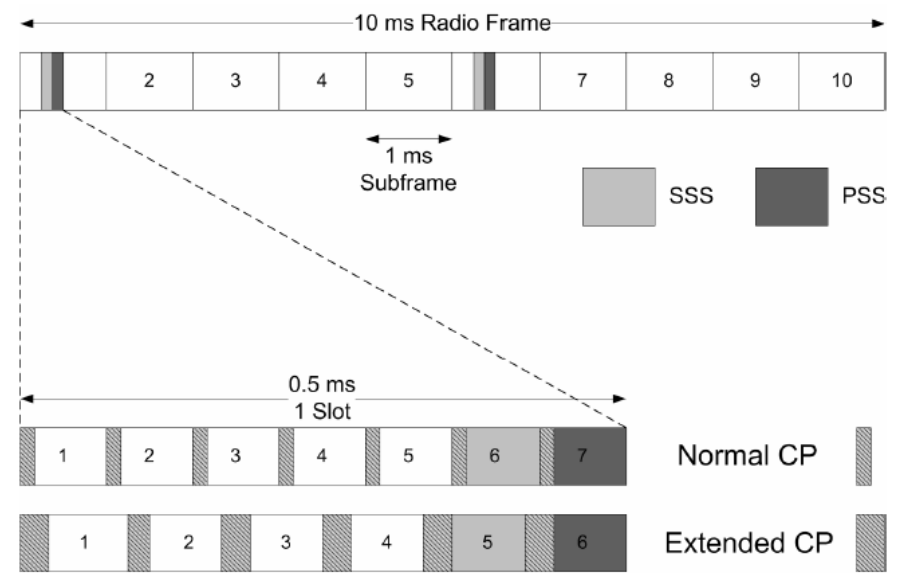

Fig. 2. PSS and SSS frame and slot structure in time domain 
PCI is calculated by using PSS and SSS in a formula (1). PCI $N_{I D}^{C E L L}$ is defined as

$$
N_{I D}^{C E L L}=3 N_{I D}^{(1)}+N_{I D}^{(2)}
$$

SSS $N_{I D}^{(1)}$ is the physical layer cell identity group (0 to 167) and PSS $N_{I D}^{(2)}$ is the identity within the group (0 to 2$)$. This arrangement creates 504 (168x3) unique physical cell identities. Next section describes PCI structure and creation of PCI signals.

\section{$2 \quad$ PSS and SSS Signal Structure}

\subsection{PSS Signal}

PSS signal consist of 3 different sequences called Physical-Layer Identities. Range is 0 to 2 and 62 symbols long. The sequence $d_{u}(n)$ used for the PSS is generated according to:

$$
\begin{aligned}
& d_{u}(n)=e^{-j \frac{\pi u n(n+1)}{63}} \text { for } n=0,1, \ldots, 30 \\
& d_{u}(n)=e^{-j \frac{\pi u(n+1)(n+2)}{63}} \text { for } n=31,32, \ldots, 61
\end{aligned}
$$

where $u$ is the Zadoff-Chu root sequence index and depends on the cell identity within the group $N_{I D}^{(2)}$ :

Table 1. PSS sequences

\begin{tabular}{|c|c|}
\hline$N_{I D}^{(2)}$ & $\begin{array}{c}\operatorname{Root} \\
\text { index } \boldsymbol{u}\end{array}$ \\
\hline 0 & 25 \\
\hline 1 & 29 \\
\hline 2 & 34 \\
\hline
\end{tabular}

\subsection{SSS Signal}

It is based on maximum length sequences (m-sequences). An m-sequence is a pseudorandom binary sequence which can be created by cycling through every possible state of a shift register of length $n$, resulting in a sequence of length $2^{\mathrm{n}}-1$. Three length-31 msequences are used to generate the synchronization signal denoted $\tilde{s}, \tilde{c}$ and $\tilde{z}$, respectively. Two length-31 binary sequences are used to generate the SSS. Sequences $s_{0}^{\left(m_{0}\right)}$ and $s_{1}^{\left(m_{0}\right)}$ are different cyclic shifts of an m-sequence $\tilde{s}$. The indices $m_{0}$ and $m_{1}$ are derived from the cell-identity group $N_{I D}^{(2)}$ and determine the cyclic shift. 


\subsection{Creating PCI}

For each cell, $\mathrm{PCI}_{\mathrm{i}}=3 \mathrm{SSS}_{\mathrm{j}}+\mathrm{PSS}_{\mathrm{k}}$

$$
\begin{aligned}
& \circ \mathrm{i}=0 \ldots 503 \\
& \circ \mathrm{j}=0 \ldots 167 \text { group } \\
& \circ \mathrm{k}=0 \ldots 2 \text { ID }
\end{aligned}
$$

The sequence for the SSS signal is generated as follows:

$$
\begin{aligned}
& \circ \mathrm{q}^{\prime}=\mathrm{INT}(\mathrm{Sj} / 30) \\
& \circ \mathrm{q}=\mathrm{INT}\left(\left(\mathrm{Sj}+\mathrm{q}^{\prime}\left(\mathrm{q}^{\prime}+1\right) / 2\right) / 30\right) \\
& \circ \mathrm{m}^{\prime}=\mathrm{S} \mathrm{j}+\mathrm{q}(\mathrm{q}+1) / 2 \\
& \circ \mathrm{m}_{0}=\mathrm{m}^{\prime} \bmod 31 \\
& \circ \mathrm{m}_{1}=\left[\mathrm{m} 0+\mathrm{INT}\left(\mathrm{m}^{\prime} / 31\right)+1\right] \bmod 31
\end{aligned}
$$

Simulations hint that the following combinations at adjacent cells will give bad performance, i.e. long synchronization times and high interference. But this is still not proven by the field tests.

- Same ID, i.e. same k

- Same $\mathrm{m}_{0}$

- Same $\mathrm{m}_{1}$

This is valid for both synchronous and asynchronous networks. Simulations for synchronous networks, shows the difference of times for camping on the network with respect to the signal to interference ratio [6].

\section{Simulation Results}

To show the synchronous time with respect to changing interference and PCI values a trial lab test was conducted. Details can be found in reference [4]. Simulation assumptions are given in reference [4], [5] and [6]. For different fading scenarios (ETU5, ETU300 and EPA5) cell identification delay computed as the time required by the UE to properly detect the cell by detecting correctly detecting its PSS, SSS and radio framing boundary [7] is illustrated in figure 3 for ETU5 where the user is mostly in this class.
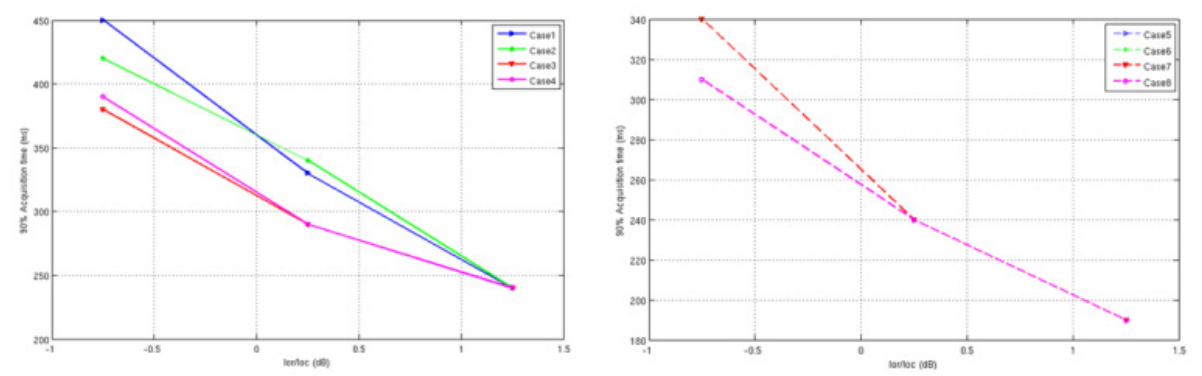

Fig. 3. Cell identification time (ETU5 channel) 
These results show that cell detection time is longer in synchronous scenario than in asynchronous scenarios for the same SNR [10]. These results show that PCI planning should have a list of approaches that needs to be defined. The following section give several recommendations.

\section{$4 \quad$ PCI Planning Recommendations}

In order to achieve the optimum quality, PCI assignment must be cell identification delay computed as the time required by the UE to properly detect the cell by correctly detecting its PSS, SSS and radio framing boundary Major recommendations about PCI planning try to prevent cells with the same Physical Cell ID from overlapping, take into consideration the neighbor cell relationships in the assignment of Physical Cell IDs, provide a method for reserving codes for use with new LTE infill sites, indoor systems, future LTE Femtocell rollout in the cell identification delay computed as the time required by the UE to properly detect the cell by detecting correctly detecting its PSS, SSS and radio framing boundary codes to be spread over distance and force a uniform use of the codes in the network [9], [4]. With respect to the above recommendations, there are two main strategy options:

1. Random planning i.e. PCI plan that does not consider PCI grouping and does not follow any specific reuse pattern

2. Ordered planning: Neighboring sites are grouped into clusters, and each cluster is assigned a limited number of Code Groups. Each site is assigned a specific Code Group and each sector a specific Color Group

Second one obviously delivers better network performance due to less interference but this method also divided into 2 subsections. One method is giving same SSS and different PSS to the cells in a site. Using the same SSS ID per site allows better management and trouble shooting in the network. This is applicable when we have a maximum of three sectors per site. It also allows optimal synchronization times in the system. This method is also advised by Ericsson because of easy use and allows optimal synchronization times [10]. Second method is more complicated. Use different PSS and also SSS. SSS values can be select with respect to different $m_{0}$ and $m_{1}$ values.[1] All together it will eliminate the risk of having the same $\mathrm{k}$ or frequency shift in the same site, in adjacent cells or pointing at each other. Clustering is another aspect to plan. Clusters can be selected equal to one Tracking Area (TA). Limitation is one TA can't exceed 28 sites. This method can be implemented for using $5 \mathrm{MHz}$ or 10 $\mathrm{MHz}$ bandwidth network, ie: For 5-10 MHz network paging load per RBS is limited and small TA is needed. Another method can be creating cluster in a TA, but then other TA borders should be carefully planned. Basic clustering and coloring showed in reference [8].

For neighboring cells PSS values are selected other than the source cell. By using WCDMA WNCS output, neighbor relation can be approximated. At the end if a cell has PSS 0, adjacent cells have PSS 1 and 2 and also best neighbor in other site have PSS 1 or 2 [9]. 


\section{Conclusion}

LTE system is novel for many networks. PCI planning is very important and any wrong assignment may cause bad experience to the user. PCI planning looks like WCDMA Scrambling Code (SC) planning. Many simulation results and papers written on it still need field experience. OFDM performs better than WCDMA by shifting some important signaling data in wide frequency band. Because of this change, PCI planning is also more complicated than SC planning [5]. When planning PCI the following priority orders are recommended:

1. The same PCI should be avoided within the same site and as neighbors.

2. PCI with conflicting $\mathrm{k}$ values should be avoided within the co-site and neighbors.

3. PCI with conflicting $\mathrm{m} 0$ and $\mathrm{m} 1$ values should be avoided within the same site and as neighbors

There is no field trial about PCI plan strategy; however the proposed way is the most optimum method to reduce interference and improve the synchronization time. If new tests show there is no or little improvement between the ordered and random method, operator can change it in more expanded network.

\section{References}

1. Holma, H., et al. (eds.): LTE for UMTS. Wiley (2009)

2. Sesia, S., et al. (eds.): LTE, The UMTS Long Term Evolution: From Theory to Practice. Wiley (2009)

3. LTE Protocols and Procedures, Ericsson (2009)

4. Performance of LTE cell identification in multi-cell environment, 3GPP TSG RAN WG4, R4-080691 (2008)

5. R4-072215, "Simulations assumptions for Intra-frequency cell identification", Texas Instru-ments, NXP, Motorola, Ericsson, Nokia

6. R4-080479, "Summary of RRM ad-hoc", Nokia Siemens Networks, Motorola

7. Ghosh, A., Zhang, J.: Fundamentals of LTE. Prentice Hall (2010)

8. LTE 10A Air Interface LZU 1087260 R1A, Ericsson (2009)

9. Kreher, R., Gaenger, K.: LT E Signaling, Troubles hooting and Optimization. John Wiley \& Sons (2011)

10. Dahlman, E., Parkvall, S.: 4G LTE/LTE-Advanced for Mobile Broadband. Elsevier (2011)

11. 3GPP Mobile Broadband Innovation Release 9, Release 10 and beyod:HSPA+, LTE/SAE and LTE Advanced (2010) 